\title{
Mealtime difficulty in older people with dementia
}

\author{
Salma Rehman1ㅡ, Gloria Likupe ${ }^{1}$, Roger Watson ${ }^{1 *}$
}

\begin{abstract}
Aim: To evaluate the evidence published in systematic reviews on the effectiveness of interventions aimed at alleviating mealtime difficulties in older people with dementia.

Background: Older people with dementia gradually lose their self-care abilities as the condition of dementia progresses and this includes the ability to eat independently. There is a large body of research into this phenomenon, including into the effectiveness of interventions to alleviate the problems which arise. Recently there have also been several systematic reviews with different conclusions about the effectiveness of these interventions.
\end{abstract}

Design: A systematic review of systematic reviews.

Methods: Databases MEDLINE, Cumulative Index to Nursing and Allied Health Literature (CINAHL), PsychINFO, Excerpta Medica Database (EMBASE), the Cochrane Library and the Joanna Briggs Library) were searched between January 2005-December 2018 using the search strategy: (feeding OR mealtimes OR eating OR intake OR food and drink OR nutrition OR difficulty) AND dementia AND intervention AND systematic review. The Critical Appraisal Skills Programme (CASP) checklist for Systematic Reviews was used to evaluate the reviews.

Results: Eight eligible studies were retrieved; three scored 10 and five scored 8 according to the CASP checklist.

Conclusion: The quality of the reviews included in the review were high. There is no strong evidence to support the use of any particular intervention for the alleviation of mealtime difficulties in older people with dementia. Methodological problems related to sample size and bias were apparent in the studies included in all the reviews and there was a lack of standardisation around interventions and outcomes across studies. Behavioural intervention, specifically Montessori education and spaced retrieval methods were considered promising and worthy of further research.

Registration: The review is registered on PROSPERO ${ }^{[1]}$

\section{Introduction}

Dementia is a syndrome describing the effect of several diseases which are common in older people. The most common type of dementia is Alzheimer's disease followed by vascular dementia, Lewy Body dementia and frontotemporal dementia. ${ }^{[2]}$ Having one type of dementia does not preclude other forms and some people have mixed. ${ }^{[2]}$ Each of these differs in aetiology and outcome but all share one feature: they adversely affect the cognitive function of the person with dementia with profound effects on memory, personality and ability to

\footnotetext{
${ }^{1}$ University of Hull, UK

*Author correspondence: r.watson@hull.ac.uk

ORCID: SR 0000-0001-9623-305X

GL $0000-0003-4524-5418$

RW 0000-0001-8040-7625

Licensed under: CC-BY

Received 15-02-2019; accepted 05-11-2019
}

live independently and carry out activities of daily living. A common problem associated with the later stages of dementia is its effect on the feeding and eating abilities of the individual. The term feeding denotes the act of moving food from a plate to the mouth ${ }^{[3][4]}$ and this is something that can be done for someone by, for example, a nurse. Eating, on the other hand, is defined as the food a person ingests. ${ }^{[5]}$ Therefore, in this article, the term 'mealtime difficulties' is used to refer to both feeding and eating difficulties as both these acts are necessary to understand and manage.

\section{Background}

The problems associated with mealtimes in older people with dementia have been well defined since the $1990 \mathrm{~s}^{[4]}$ and these include: refusal to eat; spitting out 
food; not swallowing; turning the head away; not opening the mouth; and allowing food to fall out of the mouth. However, the evidence-base for purportedly effective interventions was almost non-existent until the first decade of the present century and a review published in 2006 but reviewing articles up to $2003^{[6]}$ confirmed this and outlined the multiple problems associated with research in this area. These problems included lack of standardisation of interventions (type and duration). The range of interventions identified by Watson and Green (2006) include: altered mealtime delivery service; altered staff allocation patterns; assessment processes and altered food texture; occupational therapy interventions; verbal prompting; music; changed dining environment; and behavioural intervention. Lack of standardisation of outcomes was also evident and these included: resident participation in meals; interaction at mealtimes; weight and body mass index; self-feeding and refusal at mealtimes; eating behaviour; agitation; fluid and energy intake. Compounding the lack of standardisation of interventions and outcomes, poor designs, including: lack of randomisation; lack of blinding and small sample sizes leading to low power to detect differences were all evident. Given the fact that all studies reported positively on effective interventions, even without substantial evidence, reporting bias was probably an issue among the studies. It is notable, also, that none of the studies on mealtime difficulty to date have considered aspects associated with food and eating in different cultures. While there is a universal need to eat food for nutritional reasons, the importance of eating and mealtimes and associated practices differ greatly, for example, between Asian and western populations. In Asian culture, where food is always shared and eaten collectively, not eating may also be considered a rejection of culture. ${ }^{[7]}$ Nevertheless, eating and mealtimes also have social significance in western cultures. ${ }^{[8]}$ At the time of Watson and Green's (2006) review it was not possible to draw any conclusions about the effectiveness of any interventions. Nevertheless, since around the time of the review by Watson and $\mathrm{Green}^{[6]}$ more rigorous studies have been evident and especially since 2010 there has been a growth in better studies designed to alleviate mealtime difficulties of older people with dementia. Concomitantly, there has been a growth in systematic reviews purporting to evaluate the evidence and the purpose of this article is to conduct a systematic review of those reviews to see if any conclusions can be drawn about which interventions are effective.

\section{Literature review}

\section{Objectives}

The literature review was conducted to address the research question:

What is the evidence for the effectiveness of interventions for mealtime difficulties experienced by older adults with dementia?

\section{Review Methods}

\section{Search Methods}

A literature search using the search engines MEDLINE, Cumulative Index to Nursing and Allied Health Literature (CINAHL), PsychINFO, Excerpta Medica Database (EMBASE), the Cochrane Library and the Joanna Briggs Library was conducted to identify studies published between January 2005-December 2018. The following search strategy was used: (feeding OR mealtimes OR eating OR intake OR food and drink OR nutrition OR difficulty) AND dementia AND intervention AND systematic review.

\section{Eligibility Criteria}

To be included in this review studies had to be: (1) a systematic review; (2) written in English; (3) published in a peer reviewed journal between January 2005-December 2018; (4) explicitly using PRISMA criteria. Studies that explored feeding difficulties in older adults with dementia or in people with dementia were included. Studies that explored other aspects of mealtime behaviour exclusively, without including feeding difficulties, were excluded. Reports, case series, scholarly or theoretical papers, editorials and commentaries were excluded. The search was conducted independently by two authors (RW \& SR) who also checked the articles remaining following removal of duplicates.

\section{Quality assessment}

The articles included in the review were evaluated separately by two authors (SR \& RW) using the 10-item CASP (Critical Appraisal Skills Programme) checklist for Systematic Reviews. In the event of disagreement, we intended to use the third author. The CASP checklist was used as this is embedded within a set of seven critical appraisal tools that have been in development for over 25 years, which are now very widely used ${ }^{[9]}$ and which are internationally recognised as having utility and rigour. ${ }^{[10]}$ The results of this evaluation are shown in Table 1 with a summary of the outcomes of the reviews. 


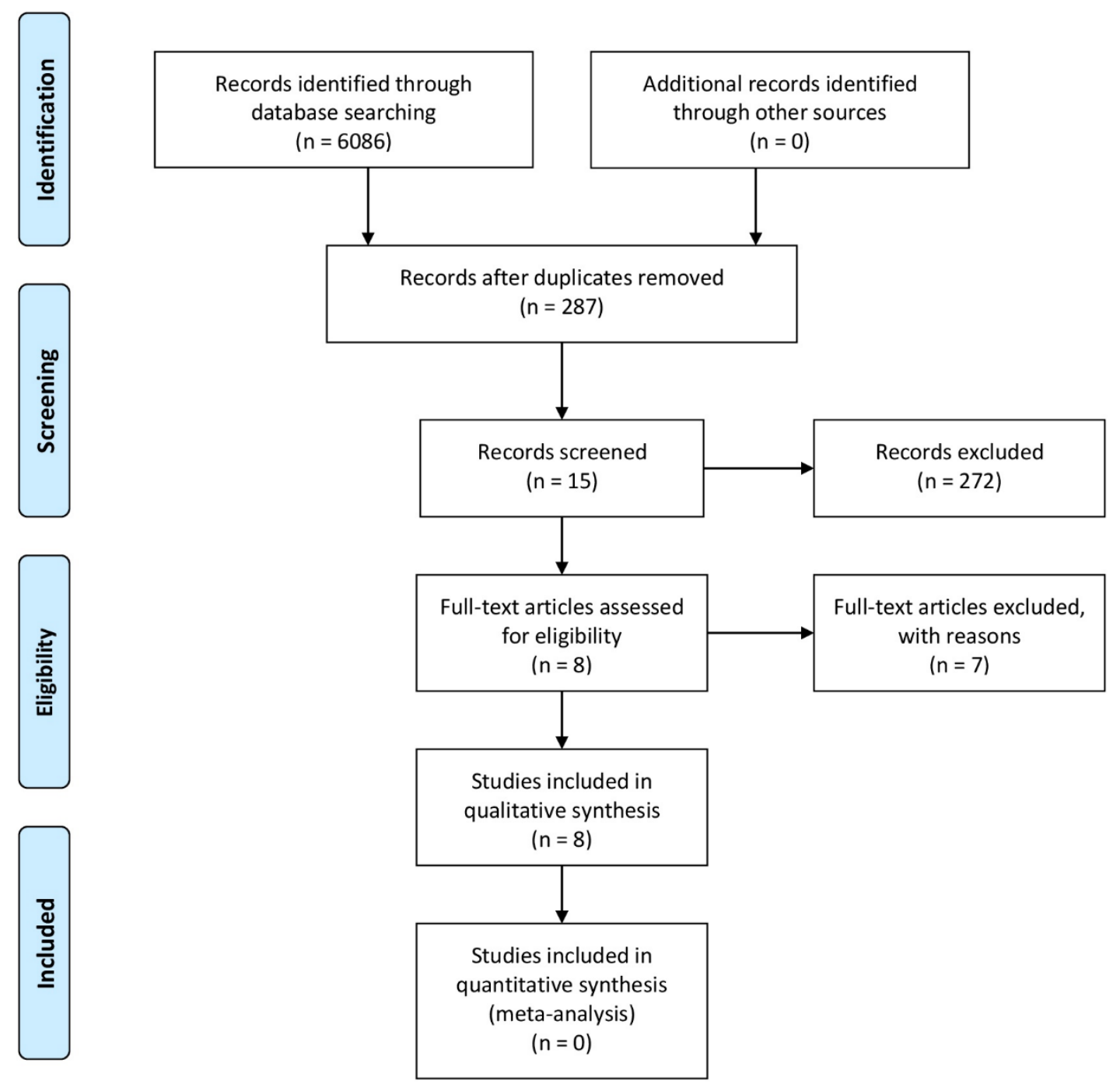

From: Moher D, Liberati A, Tetzlaff J, Altman DG, The PRISMA Group (2009). Preferred Reporting Items for Systematic Reviews and MetaAnalyses: The PRISMA Statement. PLoS Med 6(7): e1000097. doi:10.1371/journal.pmed1000097

For more information, visit www.prisma-statement.org.

Figure 1 | PRISMA flowchart

\section{Data Synthesis}

No formal method of data synthesis was applied as both the interventions and the outcomes reported in the reviews were very disparate with no two reviews having identical inclusion criteria. Therefore, data synthesis was predicated on extracting the main findings of the reviews and summarizing these in a narrative.

\section{Registration}

The review protocol is registered on PROSPERO ${ }^{[1]}$

\section{Results}

As shown in Figure 1, the initial search resulted in the identification of 6068 articles. This was reduced to 287 articles after removal of duplicates. It should be noted that $100 \%$ agreement was obtained from the initial search strategy. After screening the titles and abstracts of the 287 articles, 15 articles remained and were read, leading to the exclusion of seven further articles. Articles were removed if they were not primarily about alleviation of mealtime difficulty in dementia, for example, if they focused on micronutrients or other behavioural aspects of dementia or were related to dysphagia. Eight articles remained to be reviewed in detail. 
None of the studies defined age as such and, in fact, some of the titles of the studies, for example, three ${ }^{[11][12][13]}$ referred only to dementia. However, it was explicit in these reviews that they were referring to dementia in old age. The remaining studies were explicit about 'elderly ${ }^{[14]}$ or 'older' people being the focus of the studies and one study ${ }^{[15]}$ in which neither term was used in the title was published in a journal dedicate to studies about older people. Only one study presented information regarding age ${ }^{[16]}$ in the data extraction table indicating that the studies included people aged 62 or over.

Five reviews scored 8 out of 10 on the CASP checklist. In these five reviews the results of the studies could not be combined; therefore, the precision of the results could not be estimated using 95\% confidence intervals, which is one of the CASP criteria. Three of the reviews scored 10 out of 10 on the CASP checklist as these were both full systematic reviews with meta-analysis. Three of these (Abbot et $a_{1}{ }^{[14]}$ Abdelhamid et al ${ }^{[11]}$ \& Bunn et $\mathrm{al}^{[12]}$ ) were United Kingdom National Institute for Health Research (NIHR) funded studies and the other (Herke et $\mathrm{al}^{[13]}$ ) was a Cochrane review. None of the studies could provide strong evidence for the effectiveness of any interventions designed to alleviate mealtime difficulty for older people with dementia.

The review by Abbott et al $^{[14]}$ of 37 articles was not solely designed to investigate mealtime difficulty in older people with dementia, it included all older people living in residential homes, but this included older people with dementia and these were reported separately. Mealtime interventions were classified by Abbot et al ${ }^{[14]}$ as follows: 'changes to food service, food improvement, dining environment alteration, staff training and feeding assistance' and the outcomes studied were body weight and caloric intake. The more rigorous the studies included in the review-in other words systematic reviews with meta-analysis over observational studies-the less strong the evidence in favour of any of the interventions for either of the outcomes. Abbot et $\mathrm{al}^{[14]}$ concluded (p. 979): 'In general, most of the studies were either too small in number or too short in time to be powered to detect any change in nutritional outcome. In addition, the reporting of the studies, especially with regards to compliance of the intervention and blinding and validity of outcome measurements was poor.'

The studies by Abdelhamid et $\mathrm{al}^{[11]}$ and Bunn ${ }^{[12]}$ both emanated from the same NIHR funding stream and were conducted by the same team. The two reviews were distinguished by investigating, respectively, direct $^{[11]}$ and indirect ${ }^{[12]}$ interventions, respectively, for mealtime difficulty in older people with dementia. Direct interventions were defined ${ }^{[11]}$ as: 'interventions aimed to modify food and/or drink, provide food or drink-based supplements, provide social support, assist with eating or drinking or manage swallowing problems, alone or as part of multicomponent interventions.' (p. 3). Indirect interventions were defined ${ }^{[12]}$ as including: '... dining environment or food service modifications, educational, behavioral, exercise-type and multicomponent interventions.' (p. 3). Specific examples of direct interventions included in Abdelhamid et al's $^{[11]}$ review included: 'oral nutrition supplementation, food modification, dysphagia management, eating assistance and supporting the social element of eating and drinking.' (p. 3). Indirect interventions ${ }^{[12]}$ included: '...dining environment or food service modifications, educational, behavioral, exercise-type and multicomponent interventions.' (p. 3) Both reviews came to the same conclusion, that there was: '...no definitive evidence on effectiveness, or lack of effectiveness, of specific interventions but studies were small and short term.' However, they both listed interventions that were considered promising for a range of outcomes.

For improved hydration and eating independence there were no promising interventions but for increasing weight and improving quantity and quality of what was eaten, oral nutritional supplements and other manipulations of the diet such as dysphagia diets as well as assistance with eating were considered promising. To make mealtimes more meaningful, changes to the social element of eating such as eating with carers and family style meals were considered promising, and reminiscing about cooking could help to improve quality of life. Regarding indirect interventions, the promising interventions were also listed for a range of outcomes but nothing showed promise regarding fluid intake. For weight increase, social changes to mealtimes, music, availability of snacks, staff and caregiver education, behavioural interventions (Montessori education and spaced retrieval activities) and multicomponent interventions. To improve meaningful engagement with food, social changes, staff education and multisensory exercises were promising. Supporting eating independence may be helped by verbal prompting, behavioural interventions, and exercise. Intake of food quantity and quality may be increased by environmental changes, music, touch, bulk food services and exercise.

The review by Herke et $a^{[13]}$ is the most comprehensive and up to date review of interventions for feeding difficulty in dementia to date conducted under the auspices of the Cochrane Library. Nine studies were reviewed covering environmental and behavioural interventions. Specifically, the interventions included the provision of additional food between meals, nutritional education 
and promotion programmes, feeding skills training programmes, education programmes for nurses, and vocal and tactile feedback by carers. The outcomes included protein intake, calorie intake, body weight, and body mass index. The quality of the studies was low to moderate and, based on the review, no interventions that were effective could be identified. However, this review made some specific recommendations about future research saying that the environmental and behavioural strategies identified in the review were worth of further study, but bias in the studies had to be addressed. Blinding of participants needs to be a feature of future studies and random allocation to treatment and control groups with better description of controls. Specifically, Herke ${ }^{[13]}$ recommended: 'To establish stable changes in food and fluid intake, studies should have a follow-up of at least four days. To establish stable changes in nutritional status, studies should have a follow-up of at least 16 weeks.' and 'Participants' characteristics should be clearly described, including the type of dementia and stage of dementia, and the diagnostic criteria used.' ( $p$. 16).

Jackson et $a^{[17]}$ conducted a review under the auspices of the The Joanna Briggs Institute. Any interventions purporting to promote: 'dietary intake e.g. changes in practice of health care workers, family or volunteers, educational interventions, changes in food service' ( $p$. 1509) were included in the study and outcomes included: 'improvement in dietary intake and/or nutritional status.' (p. 1509) and this was measured by a range of parameters including body weight, body mass index, biochemical indicators and calorie intake. Eleven articles were reviewed providing only moderate support for the interventions included. In the studies the methodological problems included a lack of blinding on the single randomised trial that was included and in the other trials there was no randomisation. The remaining studies were quasi-experimental and underpowered to detect differences. Jackson also indicated that confounding variables-in particular functional ability at the start of studies-were inadequately accounted for. Jackson ${ }^{[17]}$ concluded that there was moderate support for educational intervention, changes to menus and food provision, environmental changes and increased dietetic support but that better designed studies with standardised outcome and which accounted for bias were required to provide stronger evidence.

Leah $^{[15]}$ conducted a review to: 'identify the best ways of supporting people with dementia to eat.' (p. 33) and 22 studies were included in the review. Interventions were classified as educational, environmental, assistance with eating or mixed. Outcomes were very wide including time spent eating, knowledge of nurses about eating in dementia, behaviours, weight, body mass index, eating difficulty, Mini Nutritional Assessment, eating difficulty, fluid intake, and energy intake. Leah ${ }^{[15]}$ concluded that there was only moderate evidence for environmental changes and that studies in this area showed bias through lack of randomisation. There was strong evidence for behavioural interventions (spaced retrieval and Montessori methods). Regarding educational and environmental studies, Leah ${ }^{[15]}$ concluded: 'educational studies focused the intervention on different populations, while the environmental changes were varied too, ranging from pre-meal music to back massage.' (p. 38).

Two reviews by Liv et al ${ }^{[16][18]}$ were carried out two years apart and, essentially, investigated the same phenomena. The time spans were different, but the research questions were almost identical and there was overlap between the keywords. The interventions included in the articles retrieved were: educational; mealtime/ feeding assistance; environment modification; multicomponent/mixed interventions; nutritional supplements. In Liu et al (2014) ${ }^{[16]}$ the outcomes were broad and included: '...behavioral and functional outcomes (e.g., eating time, feeding difficulties, eating ability, frequency and time of self-feeding, physical or verbal assistance/prompts, level of dependence, agitation, cognitive and behavioral function and behavioral disturbance), nutritional outcomes (e.g., food intake, body weight, BMI, nutritional status, body composition, biochemical parameters), and other adverse outcomes (e.g., occurrence of fractures, pressure ulcers and hospitalization). Caregiver outcomes included knowledge, attitude and behaviors in nursing assistants, staffing time and caregiver's burden.' (p. 18). In Liu et al $(2015)^{[18]}$ the outcomes were limited to: 'Self-feeding or eating performance (e.g., eating independence, eating frequency, eating task participation and assistance, self-feeding ability, feeding difficulty).' (Supplementary Table 3) and the following were excluded: 'enteral or parenteral nutrition; the participants were recruited from hospitals, communities, in-home-living or clinic settings; the intervention components were nutritional supplementation, nutritional education or music; the outcomes were nutritional intake, anthropometric and biochemical parameters, behavioural disturbances or other adverse events.' (p. 233) In both reviews) ${ }^{[16][18]}$ the evidence for effectiveness was generally moderate but behavioural interventions (described by Liv et al ${ }^{[16][18]}$ as training programmes) were rated as strong with nutritional supplementation and mealtime assistance rated as moderate. Problems leading to bias such as lack of blinding and randomisation were evident in the studies retrieved in both reviews. 


\section{Reference Title}

$\mathrm{R}$ Abbott et Effectiveness of mealtime interventions on $10 / 10$

al $2013^{[14]} \quad$ nutritional outcomes for the elderly living in residential care: a systematic review and meta- analysis

Abdelhamid Effectiveness of interventions to directly $10 / 10$

et al 2016 ${ }^{[11]}$ support food and drink intake in people with dementia: a systematic review

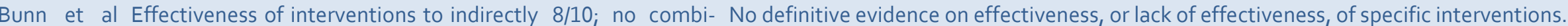

(2016) $^{[12]} \quad$ support food and drink intake in people nation of results with dementia: Eating and Drinking Well in or estimate of dementia (EDWINA) systematic review precision

Herke et al Environmental and behavioural modifica- 10/10

(2018) [13] tions for improving food and fluid intake in people with dementia (Review)

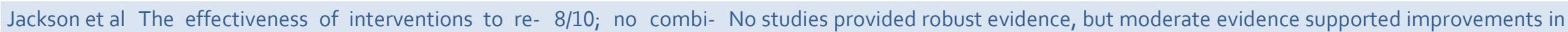

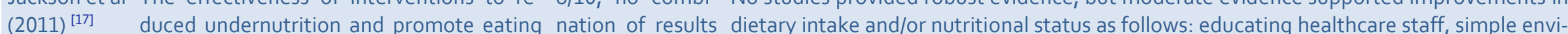
in older adults with dementia: a systematic or estimate of ronmental manipulations, for example, using high contrast tableware or small dining review precision roms, introducing something interesting, for example, an aquarium or background music, or providing feeding assistance, enhanced menus, a decentralised food service, enhanced nutritional screening with increased dietetic time.

Leah Supporting people with dementia to eat

$(2016)^{[15]}$

8/10; no combi- Strongest evidence shown in the more complex educational programmes.

nation of results

or estimate of

precision

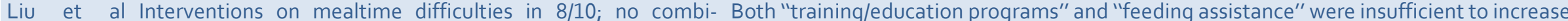
$(2014)^{[16]}$ older people with dementia: a systematic nation of results food intake. "Environment/ routine modification" indicated low evidence to increase food review or estimate of intake. precision

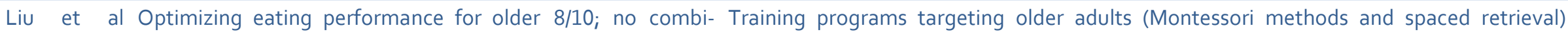

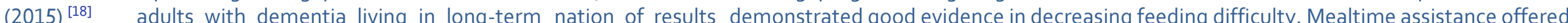
care: a systematic review or estimate of by nursing staff (e.g., verbal prompts and cues, positive reinforcement, appropriate praise precision and encouragement) also showed effectiveness in improving eating performance. 


\section{Discussion}

This study was a review of systematic reviews of interventions to improve mealtime outcomes for older people with dementia. Eight studies met the criteria for the review and were included in the analysis. Generally speaking the strength of the reviews, according to the CASP checklist for systematic reviews was strong.

Three studies ${ }^{[14][11][13]}$ fulfilled all ten criteria. The remaining five studies fulfilled eight criteria, with all eight scoring on identical criteria but lacking meta-analysis and, therefore, any estimate of the precision of the results. It is noticeable that, of the three most rigorous reviews, two were $\mathrm{NIHR}{ }^{[14][11]}$ funded and on ${ }^{[13]}$ was a full Cochrane Library review. One of the other studies ${ }^{[12]}$ was also NIHR funded but a meta-analysis of the data was not possible. Two studies ${ }^{[11][12]}$ used Cochrane Library criteria to evaluate the studies but the studies were not conducted under the auspices of the Cochrane Library. The Joanna Briggs Library process was used in one study ${ }^{[17]}$ but without meta-analysis of the data in the studies. Of the remaining studies, one ${ }^{[15]}$ was single authored and, while it fulfilled eight of the CASP criteria, there was no obvious check on the reliability of the quality assessment of studies. The remaining studies were all co-authored.

Generally, the quality of the studies included in the reviews was moderate. Across all studies the sample sizes were considered small, bias was evident due to lack of randomisation and blinding and studies were inconsistent in applying interventions. Liu et al ${ }^{[16][18]}$ did refer to some studies using behavioural interventions as being 'strong' using the Quality assessment tool for quantitative studies $^{[19]}$ (QATOS) criteria. However, these same studies, while indicated to be promising ${ }^{[12]}$ for future investigation, were not rated as strong according to the Cochrane Library criteria. The single Cochrane Library review ${ }^{[13]}$ did indicate that the environmental modification interventions and behavioural interventions were worthy of further investigation in more rigorous studies. Dietary supplementation had mixed results across the studies included in the review.

The environmental interventions and the educational interventions aimed at staff were varied with no identifiable single method being applied. However, the behavioural interventions-classified as 'indirect' by Bunn et $a^{[12]}$ - referred specifically to two methods: Montessori education and spaced retrieval activities which were the focus of two studies. ${ }^{[20][21]}$ Nevertheless, depending on the outcome-for example, feeding behaviour or food intake-these studies were rated weak to moderate using the GRADE system for evaluating studies. Specifically, the following weaknesses were pointed out by Herke et $\mathrm{al}^{[13]}$ and summarised as follows: 'Neither the Lin 2010 ${ }^{[20]}$ nor Wu 2014 ${ }^{[21]}$ studies accounted for clustering in their analyses. Where available, we used intraclass correlation coefficients (ICCS) from other sources in order to calculate a 'design effect' for these studies to reduce the effective sample size. We could not identify reliable ICCs for measures of food and fluid intake or mealtime behaviour and therefore report uncorrected measures. For these outcomes, the Cls are likely to be too narrow.' (p. 23).

\section{Conclusion}

The quality of the reviews included in this review of reviews was high, with none scoring less than 8 out of a maximum 10 points, and all of these scoring 8 on identical items. In each case this was related to the fact that the data contained within these reviews could not be aggregated. Nevertheless, studies in the area of mealtime interventions aimed at improving eating and nutritional intake are generally low in quality.

Common problems are related to design of experimental studies such as low sample sizes leading to a lack of power and bias from lack of randomisation and blinding. In the most sophisticated studies other problems such as clustering may be evident and unaccounted for. There remains a need for a stronger consensus among researchers in this field around which interventions are worthy of further study. In addition, on the one hand, there must be agreement on which outcomes are most likely to be useful and agreement around what is considered clinically effective and how long interventions should be applied so that positive effects are likely to be observed. On the other hand, the studies included in the reviews considered here all lacked economic analysis and this is an essential component to ensure that interventions which purport to work are costed properly.

The disparities around the use of terminology related to older people and the titles of articles and the lack of inclusion of age related data or explicit definitions of age in the reviews could be considered a minor weakness in these studies. Nevertheless, it may be necessary for this aspect of future studies and reviews of studies to be addressed. It is also notable, with reference to our point raised in the background section about potential cultural differences regarding mealtimes, that none of the reviews uncovered studies which considered cultural aspects of mealtimes. Currently, despite the increasing numbers of older people with dementia and the concomitant increase in the number of people who will ex- 
perience difficulties around mealtimes, the state of science in the field of research into this area cannot provide robust evidence in favour of any particular interventions.

\section{Additional information}

\section{Competing interests}

RW was the external reviewer for the Herke et al[ ${ }^{[13]}$ review, is co-author on one article ${ }^{[20]}$ related to the use of Montessori and spaced retrieval method and an Editorial Board member of the WikiJournal of Medicine.

\section{References}

1. $\uparrow$ Jump up to:1.0 1.1 "Mealtime difficulty in older people with dementia: a review of reviews". CRD PROSPERO. Retrieved 2019-02-17.

2. $\uparrow$ Jump up to:2.0 2.1 "Types of dementia". Alzheimers Society. Retrieved 2019-0207

3. $\uparrow$ Siebens, H., Trupe, E., Siebens, A., Cook, F., Anshen, S., Hanaver, R., \& Oster, G. (1986). "Correlates and consequences of eating dependency in institutionalized elderly". Journal of the American Geriatrics Society 34 (3): 192-198. doi:10.1111/j.1532-5415.1986.tb04202.x. PMID 3950287.

4. $\uparrow$ Jump up to:4.0 4.1 Watson, R. (1993). "Measuring feeding difficulty in patients with dementia: perspectives and problems". Journal of Advanced Nursing 18 (1): 25-31. doi:10.1046/j.1365-2648.1993.18010025.x. PMID 8429164.

5. $\quad \uparrow$ "Eating vs feeding". WikiDiff. Retrieved 2019-02-17.

6. $\uparrow$ Jump up to:6.0 6.1 Watson, R., \& Green, S. M. (2006). "Feeding and dementia: a systematic literature review.". Journal of Advanced Nursing 54 (1): 86-93. doi:10.1046/j.1365-2648.2006.03793.x. PMID 16553694.

7. $\uparrow$ "Eating habits in different cultures". Retrieved 2019-03-26.

8. $\uparrow$ Burges Watson, D.L., Lewis, S., Bryant, V., Patterson, J.M., Kelly, C., Edwards-Stuart, R., Muretagh, M.J., Deary, V. (2018). "Altered eating: a definition and framework for assessment and intervention". BMC Nutrition 4 (14): 192-198. doi:10.1186/s40795-018-0221-3.

9. $\quad \uparrow$ "Results for casp tools". NICE. Retrieved 2019-03-26.

10. $\uparrow$ "Critical appraisal tools to make sense of evidence". National collaborating centre for methods and tools. Retrieved 2019-03-26.

11. $\uparrow$ Jumpup to:11.0 11.111.2 11.3 11.4 11.5 11.6 11.711.811.9 Abdelhamid, A., Bunn, D., Copley, M., Cowap, V., Dickinson, A., Gray, L., Howe, A., Killett, A., Lee, J., Li, F., Polands, F., Potter, J., Richardson, K., Smithard, D., Fox, C., \& Hooper, L.
(2016). "Effectiveness of interventions to directly support food and drink intake in people with dementia: systematic review and meta-analysis". BMC Geriatrics 16 (26): 1-18. doi:10.1186/s12877-016-0196-3. PMID 26801619.

12. $\uparrow$ Jump up to:12.00 $12.01 \quad 12.02 \quad 12.03 \quad 12.04 \quad 12.05 \quad 12.06 \quad 12.07 \quad 12.08 \quad 12.0912 .10$ Bunn, D., Abdelhamid, A., Copley, M., Cowap, V., Dickinson, A., Gray, L., Howe, A., Killett, A., Poland, F., Potter, J., Richardson, K., Smithard, D., Fox, C., \& Hooper, L. (2016). "Effectiveness of interventions to indirectly support food and drink intake in people with dementia: Eating and Drinking Well IN dementiA (EDWINA) systematic review". BMC Geriatrics 16 (89): 1-21. doi:10.1186/s12877-016-0196-3. PMID 27142469.

13. $\uparrow$ Jump up to:13.0 13.1 13.2 13.3 13.4 13.5 13.6 13.7 13.8 13.9 Herke, M., Fink, A., Langer, G., Watzke, S., Nahff, A.M., \& Burckhardt, M. (2018). "Environmental and behavioural modifications for improving food and fluid intake in people with dementia". Cochrane Database of Systematic Reviews (7): 1-21. doi:10.1002/14651858.CD011542.pub2.

14. $\uparrow$ Jump up to:14.0 14.1 14.2 14.3 14.4 14.5 14.6 14.7 Abbott, R. A., Whear, R., ThompsonCoon, J., Ukoumunne, O. C., Rogers, M., Bethel, A., Gemsley, A. \& Stein, K. (2013). "Effectiveness of mealtime interventions on nutritional outcomes for the elderly living in residential care: a systematic review and meta-analysis". w:Ageing_Research_Reviews Ageing Research Reviews 12 (4): 967-981. doi:10.1016/j.arr.2013.02.002. PMID 23811415.

15. $\uparrow$ Jump up to:15.0 15.115 .215 .315 .415 .5 Leah, V. (2016). "Supporting people with dementia to eat".Nursing Older People 28 (6): 33-39. doi:10.7748/nop.2016.e811. PMID 27353791.

16. $\uparrow$ Jump up to:16.0 16.1 16.2 16.3 16.4 16.5 16.6 Liu, W., Cheon, J., \& Thomas, S. A. (2014). "Interventions on mealtime difficulties in older adults with dementia: A systematic review". International Journal of Nursing Studies 51 (1): 14-17. doi:10.1016/j.ijnurstu.2012.12.021. PMID 23340328.

17. $\uparrow$ Jump up to:17.0 17.1 17.2 17.3 Jackson, J. Currie, K., Graham, C., Robb, Y. (2011). "The effectiveness of interventions to reduce undernutrition and promote eating in older adults with dementia: A systematic review". JBI Library of Systematic Reviews 9 (37): 1509-1549. doi:10.11124/jbisrir-2011-119. PMID 27819926.

18. 个 Jump up to:18.0 18.1 18.2 18.3 18.4 18.5 Liu, W., Galik, E., Boltz, M., Nahm, E.-S., \& Resnick, B. (2015). "Optimizing Eating Performance for Older Adults With Dementia Living in Long-term Care: A Systematic Review". Worldviews on Evidence-Based Nursing 12 (4): 228-235. doi:10.1111/wvn.12100. PMID 26122316.

19. $\uparrow$ "QATQS". National Collaborating Centre for Methods and Tools. Retrieved 2019-02-08

20. $\uparrow$ Jump up to:20.0 20.1 20.2 Lin, L. C., Huang, Y. J., Su, S. G., Watson, R., Tsai, B. W. J., \& Wu, S. C. (2010). "Using spaced retrieval and Montessori-based activities in improving eating ability for residents with dementia". International Journal of Geriatric Psychiatry 25 (10): 953-959. doi:10.1002/gps.2433. PMID 20054841.

21. $\uparrow$ Jump up to:21.0 21.1 Wu, H-S., Lin, L-C., Wu, S-C., Lin, K-N., Lin, H-C. (2014). "The effects of spaced retrieval combined with errorless learning in institutionalized elders with dementia recall performance, cognitive status, and food intake". Alzheimer Disease and Associated Disorders 28 (4): 333-339. doi:10.1111/j.1365-2702.2011.03858.x. PMID 21981704. 